\title{
First application of next-generation sequencing in Moroccan breast/ovarian cancer families and report of a novel frameshift mutation of the $B R C A 1$ gene
}

\author{
FARAH JOUALI $^{1,2}$, FATIMA-ZAHRA LAARABI $^{3}$, NABILA MARCHOUDI ${ }^{1}$, ILHAM RATBI $^{3,4}$, \\ SIHAM CHAFAI ELALAOUI ${ }^{3,4}$, HOURIA RHAISSI ${ }^{2}$, JAMAL FEKKAK ${ }^{1}$ and ABDELAZIZ SEFIANI ${ }^{3,4}$
}

\begin{abstract}
${ }^{1}$ Anoual Laboratory of Radio-Immuno Analysis, Casablanca 20360; ${ }^{2}$ Laboratory of Pathophysiology and Molecular Genetics, Ben M'Sik Faculty of Science, Casablanca 7955; ${ }^{3}$ Department of Medical Genetics, National Institute of Health, Rabat 769;

${ }^{4}$ Human Genome Center, Faculty of Medicine and Pharmacy, Mohammed V University, Rabat 8007, Morocco
\end{abstract}

Received July 8, 2015; Accepted May 24, 2016

DOI: $10.3892 / 01.2016 .4739$

\begin{abstract}
At present, breast cancer is the most common type of cancer in females. The majority of cases are sporadic, but $5-10 \%$ are due to an inherited predisposition to develop breast and ovarian cancers, which are transmitted as an autosomal dominant form with incomplete penetrance. The beneficial effects of clinical genetic testing, including next generation sequencing (NGS) for BRCA1/2 mutations, is major; in particular, it benefits the care of patients and the counseling of relatives that are at risk of breast cancer, in order to reduce breast cancer mortality. BRCA genetic testing was performed in 15 patients with breast cancer and a family with positivity for the heterozygous c. $6428 \mathrm{C}>\mathrm{A}$ mutation of the BRCA2 gene. Informed consent was obtained from all the subjects. Genomic DNAs were extracted and the NGS for genes was performed using the Ion Torrent Personal Genome Machine (PGM) with a 316 chip. The reads were aligned with the human reference HG19 genome to elucidate variants in the $B R C A 1$ and BRCA2 genes. Mutations detected by the PGM platform were confirmed by target direct Sanger sequencing on a second patient DNA sample. In total, $4 B R C A$ variants were identified in 6 families by NGS. Of these, 3 mutations had been previously reported: c.2126insA of $B R C A 1$, and c.1310_1313delAAGA and c.7235insG of BRCA2. The fourth variant, c.3453delT in BRCAl, has, to the best of our knowledge, never been previously reported. The present study is the first to apply NGS of the BRCA1 and BRCA2 genes to a Moroccan population, prompting additional investigation into local founder mutations and variant characteristics in the
\end{abstract}

Correspondence to: Professor Abdelaziz Sefiani, Department of Medical Genetics, National Institute of Health, 27 IbnBatouta Avenue, Rabat 769, Morocco

E-mail: a.sefiani@um5s.net.ma

Key words: Moroccan, families, breast cancer, BRCA1, BRCA2, mutation, next generation sequencing region. The variants with no clear clinical significance may present a diagnostic challenge when performing targeted resequencing. These results confirm that an NGS approach based on Ampliseq libraries and PGM sequencing is a highly efficient, speedy and high-throughput mutation detection method, which may be preferable in lower income countries.

\section{Introduction}

At present, breast cancer is the most common type of cancer in females (1). The majority of cases are sporadic, but $5-10 \%$ are due to an inherited predisposition to develop breast and ovarian cancers, which are transmitted as an autosomal dominant form with incomplete penetrance $(2,3)$. Germline mutations of BRCA1 and BRCA2 genes are involved in $\sim 10$ and $3-5 \%$ of ovarian and breast cancers, respectively $(4,5)$. According to various professional society guidelines, BRCAI and $B R C A 2$ hereditary breast and ovarian cancer is characterised by: Multiple family members that possess breast, ovarian or both cancers; occurring at young ages or bilaterally in the case of breast cancer, triple-negative (estrogen receptor-, progesterone receptor- and human epidermal growth factor receptor 2/neu-negative) breast cancer and male breast cancer; and an increased risk of prostate, pancreatic and endometrial cancers (6,7). BRCA1 and $B R C A 2$ are tumor suppressor genes associated with DNA damage recognition, double-strand break repair, checkpoint control, transcription regulation and chromatin remodeling (8). BRACl and $B R A C 2$ are large genes containing 5,592 and 11,385 nucleotides, respectively, which spread over $\sim 100,000$ bases of genomic DNA each (9). In the Human Gene Mutation Database at the Institute of Medical Genetics in Cardiff (HGMD; http://www.hgmd.cf.ac. uk/ac/index.php), >1,833 BRCA1 mutations and 1,552 BRCA2 mutations are reported.

Clinical genetic testing for BRCA1 and BRCA2 mutations allows physicians to diagnose patients with hereditary breast and ovarian cancer. The beneficial effects of clinical genetic testing on the care and counseling of relatives at risk may aid to reduce breast cancer mortality. The results of oncogenetic services and the genetic testing of BRCA1 and BRCA2 
genes in Moroccan families by direct Sanger sequencing were previously documented by the Department of Medical Genetics of the public National Institute of Health (Rabat, Morocco) $(10,11)$. The present study reports the results of the first Moroccan investigation of BRCA1 and BRCA2 gene next generation sequencing (NGS) using an Ion Torrent Personal Genome Machine (PGM) System in 15 Moroccan patients with a family history of breast/ovarian cancer. A novel frameshift mutation of the BRCAl gene is also reported.

\section{Patients and methods}

Patients and families. The current study was performed as a collaboration between the Anoual Laboratory of Radio-Immuno Analysis, Casablanca, Morocco, where the samples were processed for NGS, and the Department of Medical Genetics of the public National Institute of Health, Rabat, Morocco, where samples were collected following oncogenetic consultations and where confirmation of the results for NGS by Sanger sequencing were performed.

Of the 68 families listed as receiving oncogenetic consultations between June 2011 and October 2015, BRCA genetic testing was performed in 15 patients with breast cancer that had been diagnosed at age 50 or younger and/or BRCA-positive family history with at least one of these criteria: Bilateral breast cancer; male relative with breast cancer; triple-negative breast cancer; ovarian, pancreatic or prostate cancer in the same individual or on the same side of the family. All subjects provided written informed consent for the study, which was performed in accordance with the Declaration of Helsinki protocols and approved by the local institutional review boards.

One positive control, who possessed the heterozygous c.6428C >A;p.Ser2143* mutation of the BRCA2 gene, was included in the molecular assay (11).

DNA extraction. Genomic DNA was isolated from peripheral blood using the salting-out method (12). The Qubit dsDNA HS (High Sensitivity) Assay kit was used to quantify DNA with the Qubit ${ }^{\circledR}$ Fluorometer (Invitrogen; Thermo Fisher Scientific, Inc., Waltham, MA, USA), according to the manufacturer's instructions.

Library preparation. A total of $10 \mathrm{ng}$ of DNA per sample was used for library preparation, using the Ion AmpliSeq ${ }^{\mathrm{TM}}$ Library kit 2.0 (Ion Torrent; Thermo Fisher Scientific, Inc.) and the Ion AmpliSeq ${ }^{\mathrm{TM}}$ BRCA1 and BRCA2 panel (Ion Torrent; Thermo Fisher Scientific, Inc.), according to manufacturer's instructions. Coding regions from $B R A C 1$ and $B R C A 2$ were amplified using 167 primer pairs for 8 samples on one Ion $316^{\mathrm{TM}}$ Chip kit v2 (Ion Torrent; Thermo Fisher Scientific, Inc.). Each library was barcoded using Ion Xpress ${ }^{\mathrm{TM}}$ Barcode Adapters kit (Ion Torrent; Thermo Fisher Scientific, Inc.).

Template preparation by emulsion polymerase chain reaction (PCR). The Qubit ${ }^{\circledR}$ 2.0 Fluorometer (Invitrogen; Thermo Fisher Scientific, Inc.) and the associated High Sensitivity DNA kit (Invitrogen; Thermo Fisher Scientific, Inc.) were used to determine the quality and concentration of the libraries. Barcoded libraries were pooled and diluted to a final concentration of $100 \mathrm{pM}$. Template preparation by emulsion PCR was performed using the Ion PGM ${ }^{\text {TM }}$ Template OT2 200 kit, followed by the Ion OneTouch ${ }^{\mathrm{TM}} 2$ System (Ion Torrent; Thermo Fisher Scientific, Inc.), according to manufacturers' protocol.

Quantification of the recovered particles was performed using the Qubit 2.0 ${ }^{\circledR}$ Fluorometer, and the Ion Sphere Quality Control kit (Ion Torrent; Thermo Fisher Scientific, Inc.) was used to evaluate the library quality, according to manufacturer's protocol. The optimal amount of template-positive ion sphere particles (ISPs) is 10-30\%. ISPs were then recovered and enriched for template positive ISPs using Dynabeads MyOne Streptavidin C1 beads (Invitrogen; Thermo Fisher Scientific, Inc.) in the Ion OneTouch ${ }^{\mathrm{TM}} \mathrm{ES}$ instrument (Ion Torrent; Thermo Fisher Scientific, Inc.).

Ion Torrent PGM sequencing. Sequencing primer and polymerase were added to the final enriched ISPs prior to loading onto an Ion 316 chip, according to the Ion PGM ${ }^{\mathrm{TM}} 200$ sequencing kit protocol. Sequencing was performed on the PGM (Ion Torrent; Thermo Fisher Scientific, Inc.). For a sequence variant to be considered authentic, a sequencing coverage of $250 \mathrm{X}$ reads was used as a minimum requirement in the present study.

Data analysis. Sequence data were processed using the Torrent Suite software v3.6 (Ion Torrent; Thermo Fisher Scientific, Inc.) to parse barcode reads, to align reads to the HG19 reference genome and to generate run metrics, including chip loading efficiency and total read counts and quality.

Coverage analysis and variant calling used Torrent Variant Caller plugin software v3.6 in the Torrent Server (http://129.130.90.13/ion-docs/Torrent-Variant-Caller-Plugin. html). The variant caller parameter setting was germline PGM high stringency. Following data analysis, annotation of single-nucleotide variants, insertions, deletions and splice site alterations was performed using the Ion Reporter Server System (Thermo Fisher Scientific, Inc.).

All mutations reported were confirmed in the Breast Cancer Information Core (BIC) database (http://www. research.nhgri.nih.gov/bic/), the HGMD (http://www.hgmd. cf.ac.uk/ac/index.php), Clinvar (http://www.ncbi.nlm.nih.gov/ clinvar/) and previous publications (10,13-15). Amino acid predictions were performed using the scale-invariant feature transform algorithm and PolyPhen-2 software tools (Polymorphism Phenotyping v2.2.2, developed by Dr Shamil Sunyaev, Harvard Medical School, Boston, MA, USA; http://genetics. bwh.harvard.edu/pph2/).

Sanger sequencing. Mutations detected by the PGM platform were confirmed by target direct Sanger sequencing. Sanger sequencing was performed using the BigDye Terminator v3.1 Cycle Sequencing kit (Applied Biosystems; Thermo Fisher Scientific, Inc.). The purification of sequencing reactions was performed using the Sephadex ${ }^{\circledR}$ (Sigma-Aldrich, St. Louis, MO, USA) method. The samples were run on an ABI 3130 Genetic Analyzer (Applied Biosystems; Thermo Fisher Scientific, Inc.). The chromatograms of the Sanger sequencing results were analyzed using SeqScape software version 2.5 (Applied Biosystems; Thermo Fisher Scientific, Inc.). 

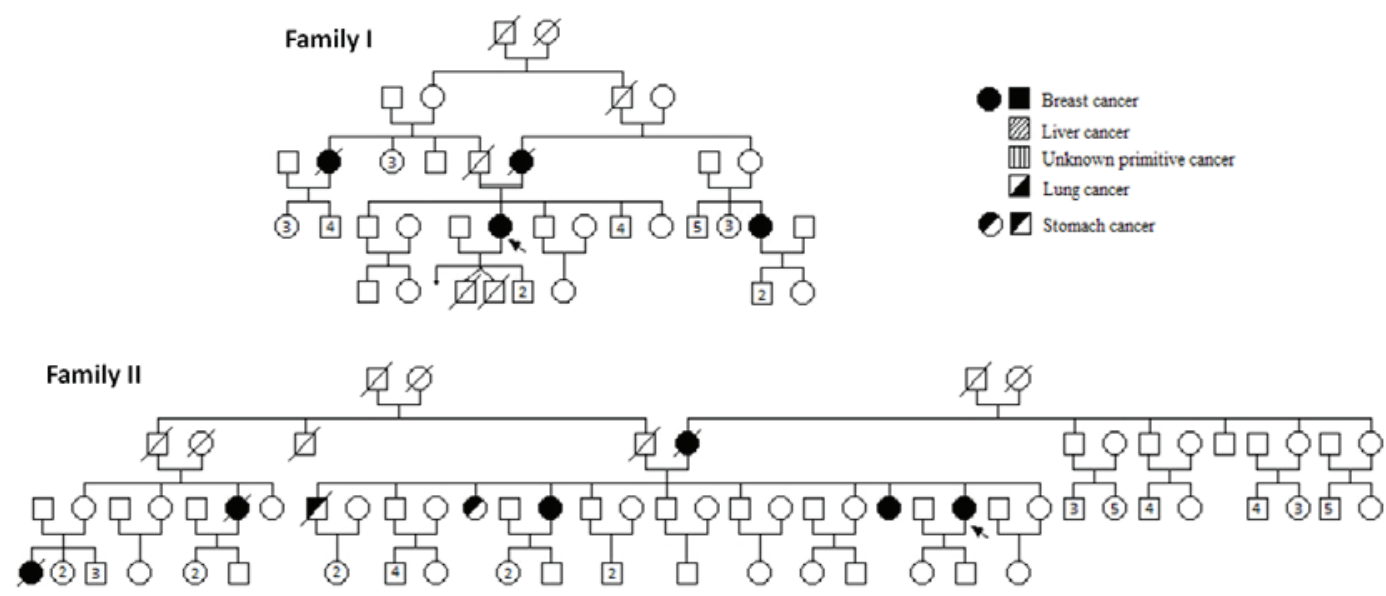

Family III

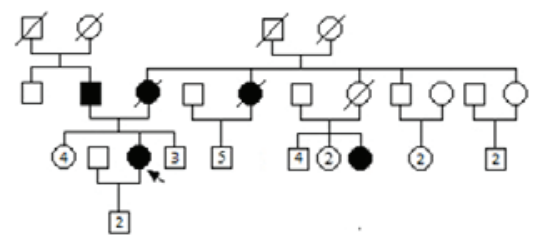

Family IV
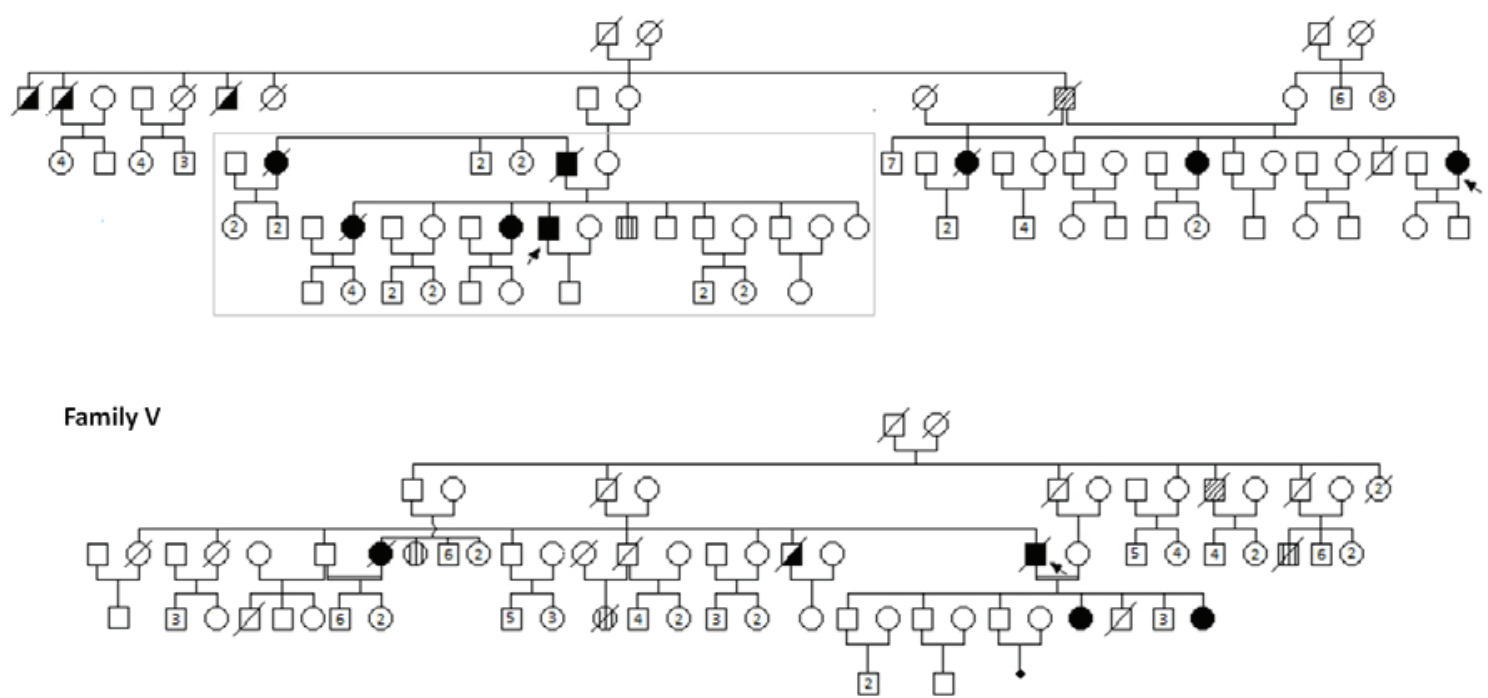

Figure 1. Pedigrees of the families with $B R C A 1$ and $B R C A 2$ mutations. Arrows indicate the two individuals (probands) in whom next generation sequencing was undertaken and blood was analysed. The proband and members of family IV in a sidebar (referred as surrounded branch) were first investigated separately in a genetic consultation. Upon genealogic investigation, it was revealed that they shared a common ancestor with the other proband and members of the unsurrounded branch. The individuals did not know that they belong to the same family. Genetically, they are considered as a unique family. The nomenclature of genealogic pedigrees is represented according to Bennett et al (21).

\section{Results}

In total, 4 BRCA mutations were identified in 6 Moroccan families. The pedigrees of mutated families are shown in Fig. 1. In the BIC database, 2 reported mutations were found in families I and II, the c.2126insA;p.Phe709TyrfsX3 mutation in exon 11 of BRCA1 and the c.7234_7235insG;p.Thr2412Serfs mutation in exon 14 of $B R C A 2$, respectively. The proband of family III was heterozygous for a BRCA1 nucleotide c.3453delT mutation in exon 11. This frameshift changes amino acid 1,151 from aspartic acid to glutamic acid, which results in a premature stop codon at amino acid 4. To the best of our knowledge, this mutation had never been reported in the BIC database, or other resources. The two protein prediction programmes, used to predict the functional consequence of this BRCAI p.Asp1151Glufs mutation, estimated it to be pathogenic. The fourth c.1310_1313delAAGA;p.Lys437IlefsX22 mutation, already listed in the BIC, was identified in 3 apparently unrelated patients, all from the North-East of Morocco. After detailed genealogic investigations, 2 of the patients were found to share a common ancestor in the second generation (family IV), whereas the third patient was not related to them for at least five generations and belonged to an independent family (family V).

NGS confirmed that the positive control was heterozygous for the mutation c. $6428 \mathrm{C}>\mathrm{A}$;p.Ser $2143^{*}$ in the BRCA2 gene. 


\section{Discussion}

Approximately $10 \%$ of ovarian cancer cases and 3-5\% of breast cancer cases are due to germline mutations in $B R C A 1 / 2(4,5)$. The mutational spectrum of $B R C A 1 / 2$ in the Moroccan population is becoming partially characterised thanks to local genetic centers, who have developed oncogenetic consultations for familial forms of breast and ovarian cancers and performed molecular analyses of BRCA genes using the conventional individual exon-by-exon Sanger sequencing $(10,13,14,16,17)$. Thus, several mutations in two particular genes have been reported in the Moroccan National Genetic Database (http:// ethnos.findbase.org/home-ma). The mutations are distributed throughout the coding region and flanking intronic sequences, with an increased concentration in exon 11 for the two genes. The majority of the mutations are frameshifts, resulting in a frameshift reading of nonsense mutations or splice site alterations that lead to truncated proteins. Of these, only the c.66_67delAG (previously c.185delAG) and c.798_799delTT mutations of BRCAl appear to be recurrent, at least in two unrelated patients $(10,15)$. In addition, the c.66_67delAG mutation has also been detected twice at the Department of Medical Genetics of the public National Institute of Health at Rabat (unpublished data).

Mutation c.798_799delTT has been observed in two Algerian and two Tunisian families presenting with breast cancer and is the first non-Jewish founder mutation to be described in Northern Africa, whereas the other identified mutations are unique $(10,11,15)$. This knowledge allows Moroccan females that belong to families with an increased risk of developing breast cancer to benefit from a presymptomatic diagnosis of the known familial causative mutation, and receive preventive management (10). One of the major constraints of conventional Sanger sequencing, despite being the gold standard for mutation screening for several years, is that it is expensive to perform and is time consuming; even more so for testing large genes such as $B R C A 1 / 2$, with limited funding and the absence of a targeted molecular testing strategy. The advent of NGS technology has increased sequencing capacity and lowered the cost of sequencing, thus offering a powerful alternative to Sanger sequencing for genetic testing (18). In addition, the launch of benchtop sequencers with the capacity to detect mutations from a significantly greater number of samples in parallel, and in a more cost effective manner, led to more clinical laboratories adopting this technology $(19,20)$.

The present study reports a family-based approach using an Ion Torrent PGM System to detect mutations in all exons of $B R C A 1 / 2$ genes in 15 patients. The approach found causative mutations in 6/14 unrelated families (43\%). This high rate of detection is associated with the selective criteria used in the present study to enroll patients into the cohort. In particular, a novel frameshift c.3453delT mutation was identified in exon 11 of the $B R C A 1$ gene, and is predicted to be deleterious in silico, according to the results of the scale-invariant feature transform algorithm and PolyPhen-2 software tools used in the present study. This finding will enrich the Moroccan National Genetic Database and other resources. In addition to the two previously reported mutations (c.185delAG and c.798_799delTT) with repeated occurrence $(10,15)$, a third mutation (c.1310_1313delAAGA;p.Lys437IlefsX22) of BRCA1 was identified in two families from the North-East of Morocco in the present study. Further investigation into these three mutations is recommended to be conducted on a larger cohort of patients to evaluate their distribution in Morocco. This information may simplify genetic testing and have clinical and public health implications. Targeted high-throughput NGS may therefore be an effective method to identify mutations for disease-affected pedigrees. Due to the large number of calling variants present in the NGS assays, genetic expertise is required to validate these variants prior to confirmation by Sanger and for use in medical practice. Patients without an identified mutation in the $B R C A 1 / 2$ genes may be explained by large rearrangements that are not detected by NGS or by mutations of other unknown genes that predispose women to breast cancer.

To the best of our knowledge, this is the first Moroccan study to perform $B R C A 1 / 2$ genetic sequencing using NGS methods in 15 Moroccan breast cancer patients with a family history. Among a wide range of variants detected in the 2 genes, 4 deleterious mutations, including a novel and recurrent mutation of $B R C A 1$, were identified. These results support that the NGS approach based on Ampliseq libraries and Ion Torrent PGM sequencing, despite the error rates in homopolymer stretches, is a highly efficient, fast and cheap high-throughout mutation detection method that may be integrated into the public health program for the molecular testing of $B R C A$ genes. The method may result in the better management of patients and genetic counseling of relatives at risk.

\section{References}

1. Willems PJ: Susceptibility genes in breast cancer: More is less? Clin Genet 72: 493-496, 2007.

2. Lynch HT, Albano WA, Danes BS, Layton MA, Kimberling WJ Lynch JF, Cheng SC, Costello KA, Mulcahy GM, Wagner CA, et al: Genetic predisposition to breast cancer. Cancer 53: 612-622, 1984.

3. Claus EB, Schildkraut JM, Thompson WD and Risch NJ: The genetic attributable risk of breast and ovarian cancer. Cancer 77: 2318-2324, 1996.

4. Robson ME, Boyd J, Borgen PI and Cody HS IIIrd: Hereditary breast cancer. Curr Probl Surg 38: 387-480, 2001.

5. Risch HA, McLaughlin JR, Cole DE, Rosen B, Bradley L, Fan I, Tang J, Li S, Zhang S, Shaw PA and Narod SA: Population BRCA1 and BRCA2 mutation frequencies and cancer penetrances: A kin-cohort study in ontario, canada. J Natl Cancer Inst 98: 1694-1706, 2006.

6. Culver J, Lowstuter K and Bowling L: Assessing breast cancer risk and BRCA1/2 carrier probability. Breast Dis 27: 5-20, 2006.

7. Evans DG and Howell A: Breast cancer risk-assessment models. Breast Cancer Res 9: 213, 2007.

8. Mohamad HB and Apffelstaedt JP: Counseling for male BRCA mutation carriers: A review. Breast 17: 441-450, 2008.

9. Filippini S, Blanco A, Fernández-Marmiesse A, Alvarez-Iglesias V, Ruíz-Ponte C, Carracedo A and Vega A: Multiplex SNaPshot for detection of BRCA $1 / 2$ common mutations in spanish and spanish related breast/ovarian cancer families. BMC Med Genet 8: 40, 2007.

10. Laarabi FZ, Jaouad IC, Ouldim K, Aboussair N, Jalil A, Gueddari BE, Benjaafar N and Sefiani A: Genetic testing and first presymptomatic diagnosis in moroccan families at high risk for breast/ovarian cancer. Oncol Lett 2: 389-393, 2011

11. Guaoua S, Ratbi I, Lyahyai J, El Alaoui SC, Laarabi FZ and Sefiani A: Novel nonsense mutation of BRCA2 gene in a Moroccan man with familial breast cancer. Afr Health Sci 14: 468-471, 2014. 
12. Miller SA, Dykes DD and Polesky HF: A simple salting out procedure for extracting DNA from human nucleated cells. Nucleic Acids Res 16: 1215, 1988.

13. Tazzite A, Jouhadi H, Nadifi S, Aretini P, Falaschi E, Collavoli A, Benider A and Caligo MA: BRCA1 and BRCA2 germline mutations in Moroccan breast/ovarian cancer families: Novel mutations and unclassified variants. Gynecol Oncol 125: 687-692, 2012.

14. Tazzite A, Nadiffi S, Kottwitz D, El Amrani M, Jouhadi H, Benider A, Moumen A and Sefrioui H: Specific BRCA1 gene variations amongst young Moroccan breast cancer patients. Genet Mol Res 13: 791-798, 2014.

15. Laraqui A, Uhrhammer N, Rhaffouli HE, Sekhsokh Y, Lahlou-Amine I, Bajjou T, Hilali F, El Baghdadi J, Al Bouzidi A, Bakri Y, et al: BRCA genetic screening in Middle Eastern and North African: Mutational spectrum and founder BRCA1 mutation (c.798_799delTT) in North African. Dis Markers 2015: $194293,2015$.

16. Elalaoui SC, Jaouad IC, Laarabi FZ and Elgueddari Bel K: Low level of consanguinity in Moroccan families at high risk of breast cancer. Asian Pac J Cancer Prev 14: 723-726, 2013.
17. Laraqui A, Uhrhammer N, Lahlou-Amine I, El Rhaffouli H, El Baghdadi J, Dehayni M, Moussaoui RD, Ichou M, Sbitti Y, Al Bouzidi A, et al: Mutation screening of the BRCA1 gene in early onset and familial breast/ovarian cancer in Moroccan population. Int J Med Sci 10: 60-67, 2013.

18. Baker M: Next-generation sequencing: Adjusting to data overload. Nature Methods 7: 495-499, 2010.

19. Chan M, Ji SM, Yeo ZX, Gan L, Yap E, Yap YS, Ng R, Tan PH, Ho GH, Ang P and Lee AS: Development of a next-generation sequencing method for BRCA mutation screening: Acomparison between a high-throughput and a benchtop platform. J Mol Diagn 14: 602-612, 2012.

20. Costa JL, Sousa S, Justino A, Kay T, Fernandes S, Cirnes L, Schmitt F and Machado JC: Nonoptical massive parallel DNA sequencing of BRCA1 and BRCA2 genes in a diagnostic setting. Hum Mutat 34: 629-635, 2013.

21. Bennett RL, French KS, Resta RG and Doyle DL: Standardized human pedigree nomenclature: Update and assessment of the recommendations of the National Society of Genetic Counselors. J Genet Couns 17: 424-433, 2008. 